\title{
Perceptual Categorization of Cat and Dog Silhouettes by 3- to 4-Month-Old Infants
}

\author{
Paul C. Quinn \\ Washington \& Jefferson College \\ and \\ Peter D. Eimas and Michael J. Tarr \\ Brown University
}

\begin{abstract}
Given evidence that silhouette information can be used by adults to form categorical representations at the basic level, four experiments utilizing the familiarization-novelty preference procedure were performed to examine whether 3- and 4-month-old infants could form categorical representations for cats versus dogs from the perceptual information available in silhouettes (e.g., global shape and external outline). Experiments 1 and 2 showed that infants could form individuated categorical representations for cat and dog silhouettes, whereas Experiments 3 and 4 revealed that infants could use silhouette information from the head, but not the body, to categorically separate the two species. These results indicate that general shape or external contour information that is centered about the head is sufficient for young infants to form individuated categorical representations for cats and dogs. The data thus provide information regarding the nature of the perceptual information that can be used by infants to form category representations for individual animal species and are discussed in terms of domain-general versus domain-specific processing accounts. () 2001 Academic Press

Key Words: categorization; visual cognition; perceptual development; cognitive development.
\end{abstract}

Perceptual categorization refers to the recognition of discriminably different entities as members of the same category based on some internal representation of the category (e.g., Edelman, 1987). In this article, the term categorical representation is used to describe this internal representation. When and how categorical representations are initially formed, elaborated, integrated with other knowl-

This research was supported by Grant HD-28606 from the National Institute of Child Health and Human Development to PDE and PCQ and by Grant BCS-0096300 from the National Science Foundation to PCQ. We thank June Shepp, Laurie Yarzab, and Patrice Pop for their assistance in testing infants and analyzing data.

Address correspondence and reprint requests to Paul C. Quinn, Department of Psychology, Washington \& Jefferson College, 60 South Lincoln Street, Washington, PA 15301. E-mail: pquinn@ washjeff.edu. 
edge structures (i.e., concepts), and related to language have been critically important issues for contemporary experimentalists and theorists (e.g., Gelman, 1996; Goldstone \& Barsalou, 1998; Jones \& Smith, 1993; Madole \& Oakes, 1999; Malt, 1995; Mandler \& McDonough, 1998; Millikan, 1998; Quinn \& Eimas, 1997; Rakison \& Butterworth, 1998; Schyns, Goldstone, \& Thibaut, 1998; Thelen \& Smith, 1994; Waxman \& Markow, 1995; Xu \& Carey, 1996). Here we continue an inquiry into the nature of the perceptual information that infants use to form categorical representations for individual animal species (Eimas \& Quinn, 1994; Eimas, Quinn, \& Cowan, 1994; Quinn \& Eimas, 1996a; Quinn, Eimas, \& Rosenkrantz, 1993; Spencer, Quinn, Johnson, \& KarmiloffSmith, 1997).

A technique that has been used to examine perceptual categorization in young infants is the familiarization-novelty preference procedure, which is based on the established preference that infants from birth onward show for novel stimuli (Fantz, 1964; Slater, 1995). This procedure involves presentation of a number of paired exemplars from a common category, followed by presentation of a novel exemplar from the familiar category paired with a novel exemplar from a novel category. If infants generalize their familiarization to novel exemplars from the familiar category, and display a preference for novel members of the novel category, then it can be inferred that the infants have formed a categorical representation of the familiar category exemplars and have perceived the novel exemplars from the novel category to be noninstances of the familiar category. This inference is also contingent on the results of control experiments showing that the pattern of preferences cannot be attributed to (1) a failure to discriminate among the instances comprising the familiar category or (2) an a priori preference for the novel category exemplars.

In using the term categorical representation to account for the looking behavior of young infants, we do not mean to imply that infants understand the meaning of the category, that the category has a rigid boundary, or that the category has a structuring entity (i.e., a prototype) or a name. Indeed it is possible to argue that these representations are not categories as commonly understood, but rather the primitive similarity-based forerunners of the categories of early childhood and beyond. While we take these representations to be categorical in nature, we recognize that all that can be inferred from existing data is that the familiar members of the category have in some manner been grouped together in an $n$-dimensional psychological space and that this cluster representation excludes novel members of novel categories. Novel members of the familiar category are responded to as being more similar to the representation of the set of familiar category members than are novel category members. Infant performance could be based on a simple exemplar model of perceptual categorization in which a boundary between categories is not present (Anderson, 1995; Quinn \& Eimas, 1998). During categorization of an exemplar, it is compared with the mentally represented nearest neighbor along a number of dimensions in psychological space that define or at least represent the exemplar under consideration. If the exemplar meets the criterion for 
similarity with its nearest neighbor, it is assigned the categorical designation to which the nearest neighbor belongs; if not, formation of a new categorical representation is begun. This is all we mean by the term categorical representation.

Recent evidence indicates that the categorical distinctions among animal species made by young infants are often the same distinctions that later in life come to have a conceptual nature in a domain of knowledge organized by a naive theory of biology (Quinn \& Eimas, 1997). For example, 3- and 4-month-olds familiarized with realistic color photographs of domestic cats generalized their familiarization to novel domestic cats, but displayed novel category preferences for birds, dogs, horses, and tigers. This finding indicates that young infants can form a categorical representation for domestic cats that includes novel domestic cats, but excludes birds, dogs, horses, and tigers.

Positive evidence for categorization of animal species by infants raises questions about the types of information and kinds of processes used to form the underlying categorical representations. The young age of the infants and the fact that the stimuli were presented as silent, static, noninteracting photographs lead one to infer that perceptual attributes that can be detected from the surfaces of the stimulus photos are the likely basis. For example, it seems improbable that young infants' categorical organization of visual patterns was guided by word (i.e., verbal label) cues, as may be the case in older infants (Balaban \& Waxman, 1997; Waxman \& Markow, 1995), or more conceptual attributes like self-initiated motion (e.g., Mandler \& McDonough, 1993), or even less obvious information regarding the biological or cognitive status of the animals (Carey, 1985; Keil, 1989; Wellman, 1990). Thus, infants might categorically differentiate cats from birds on the basis of the number of legs, cats from horses on the basis of overall shape, and cats from tigers on the basis of surface markings.

An issue of interest has been how young infants categorically separate animal species that bear a close resemblance to each other, such as cats and dogs (Quinn \& Eimas, 1996b). These two species are similar to each other in terms of the presence of fur, the quality of their coloring, the number of legs they possess, the nature of their facial features, and the overall shape and size of their bodies. One could reason that subtle differences in (1) any one attribute, (2) the pattern of correlation across a number of the attributes, or (3) the overall gestalt might be the basis for a perceptual partitioning of the two categories (Malt \& Smith, 1984; Marr, 1982; Murphy, 1991; Rosch, Mervis, Gray, Johnson, \& Boyes-Braem, 1976; Tversky \& Hemenway, 1984). Of particular relevance is research indicating that certain forms of contour information play an important role in perceptual categorization in adults. For example, there is both theory and evidence indicating that object silhouettes can support perceptual categorization (e.g., Hayward, 1998; Kurbat, Smith, \& Medin, 1994; Rosch et al., 1976). Moreover, computational models of silhouette similarity have demonstrated that object silhouettes cluster into groups that correspond quite closely to what human observers consider to be basic-level categories (Cutzu \& Tarr, 1997; Gdalyahu \& Weinshall, 1996; Ullman, 1996). 
In a previous investigation, we found that information centered about the face and head provided a sufficient, and possibly necessary, basis for young infants' categorical representation of cats and dogs as separate from each other (Quinn \& Eimas, 1996a). Three- and 4-month-olds familiarized with cat heads preferred a novel dog head over a novel cat head; however, infants of the same age familiarized with cat bodies divided their attention evenly between novel dog and novel cat bodies. It was further shown that the infants' ability to use head and face information generalized to considerably enlarged cat heads presented in isolation and that the cues for this categorical representation resided in the external surface area of the head (i.e., the region outside the configuration of internal facial features) and in the internal facial region inclusive of the eyes, nose, and mouth.

The importance of the face and head region was corroborated in a study in which infants familiarized with cats or dogs preferred a hybrid animal consisting of a novel category head and familiar category body over a hybrid animal consisting of a novel category body and familiar category head (Spencer et al., 1997). Also consistent with the empirical findings indicating that the head and face region is critical for young infants' categorical differentiation of cats and dogs are simulation results from simple neural networks that failed to learn individuated representations for cats and dogs when provided with inputs reflecting only the bodily attributes (and not the head and face features) of the animal stimuli (Quinn \& Johnson, 1997).

The focus of the current research is the nature of the perceptual information that can be used by infants to segregate cats and dogs into differentiated classes. We report a series of experiments that attempt to extend our major findings obtained previously with realistic pictures of cats and dogs to cat and dog silhouettes. The results are relevant to domain-general and domain-specific processing accounts of early cognitive development. From a domain-general perspective, the outline of the head region may be the most differentiated part of the stimulus that is diagnostic of category membership. Consequently, any generic pattern learning mechanism would come to rely on this region for the categorical partitioning of cats and dogs and, presumably, other animal species. Alternatively, from a domain-specific perspective, it is possible that infants are born with a mechanism that orients them to the head and face region of the stimulus. One such mechanism, called CONSPEC by Johnson and Morton (1991), has been proposed to account for how various species recognize conspecifics. It has been hypothesized that CONSPEC operates on the basis of a predefined schematic representation of the eyes, nose, and mouth, in their normal configuation-a kind of innate face prototype. It is possible that in human infants CONSPEC might use a face representation that is sufficiently general so as to be able to differentiate cat and dog faces. It is also possible that a mechanism like CONSPEC could be reformulated to include information about external head shape. Thus, infants' ability to categorically differentiate between dog and cat silhouettes could be based on more general pattern learning mechanisms sensitive to the information in the external 
contour of the head region or to more specialized pattern learning mechanisms sensitive to head shape or both.

\section{EXPERIMENT 1}

Infants were presented with silhouettes of the pictures of cats and dogs used in earlier experiments to determine whether the bounding contour information in these uniformly black pictures provided sufficient information to permit the formation of categorical representations. Three- to 4-month-olds were familiarized with a set of 12 silhouettes of cats or dogs and then given two test trials pairing novel dog and cat silhouettes.

\section{Method}

Participants. Participants were 48 healthy, full-term 3- to 4-month-old infants ( $M=3.33$ months; $S D=.22$ months). Twenty-two infants were female and 26 were male. One additional infant did not complete the experiment because of fussiness.

Stimuli. The stimuli consisted originally of 18 pictures of dogs and 18 pictures of cats, the specifics of which were described in Quinn et al. (1993) and Quinn and Eimas (1996a). The naturally appearing pictures of the dogs and cats were transformed into silhouettes of exactly the same size, but in a uniform black color. Each picture contained a single animal that was centered and pasted onto a white $17.7 \times 17.7-\mathrm{cm}$ posterboard for presentation. Examples of two cat and dog photographs and their corresponding silhouettes are shown in Figs. 1 and 2. ${ }^{1}$

Apparatus. All infants in each experiment were tested in a visual preference apparatus, modeled on the one described by Fagan (1970). The apparatus is a large, three-sided gray viewing chamber that is on wheels. It has a hinged, gray display panel onto which were attached two compartments to hold the posterboard stimuli. The stimuli were illuminated by a fluorescent lamp that was shielded from the infant's view. The center-to-center distance between compartments was $30.5 \mathrm{~cm}$ and on all trials the display panel was situated approximately 30.5 $\mathrm{cm}$ in front of the infant. There was a $0.625-\mathrm{cm}$ peephole located midway between the two display compartments that permitted an observer to record the infant's visual fixations.

${ }^{1}$ In Quinn et al. (1993), we found that categorical representations for dogs were more readily established if the exemplars were more typical members of the category than if the typicality scores were lower and more variable. To prevent differences in variability between the cats and dogs from being at issue in subsequent studies (e.g., Quinn \& Eimas, 1996a), the four most atypical dogs in the stimulus set were replaced by four dogs judged to be more typical. To be sure that there were no differences in the variability in the cat and dog silhouettes in the present study, we showed the two sets of silhouettes (i.e., the cats and reduced variability set of dogs) to 20 mothers who brought their infants to the laboratory. They were asked to rate each of the 36 silhouettes on a scale from 1 to 9 , with 1 defined as $a$ very poor example of a cat or dog, 5 as a moderately good example, and 9 as a very good example. The two groups did not differ in the mothers' ratings of typicality, Dogs: $M=6.41, S D=2.28$; Cats: $M=6.20$, $S D=2.38 ; t(19)=1.59, p>.10$, and thus no asymmetry in categorization was expected. 

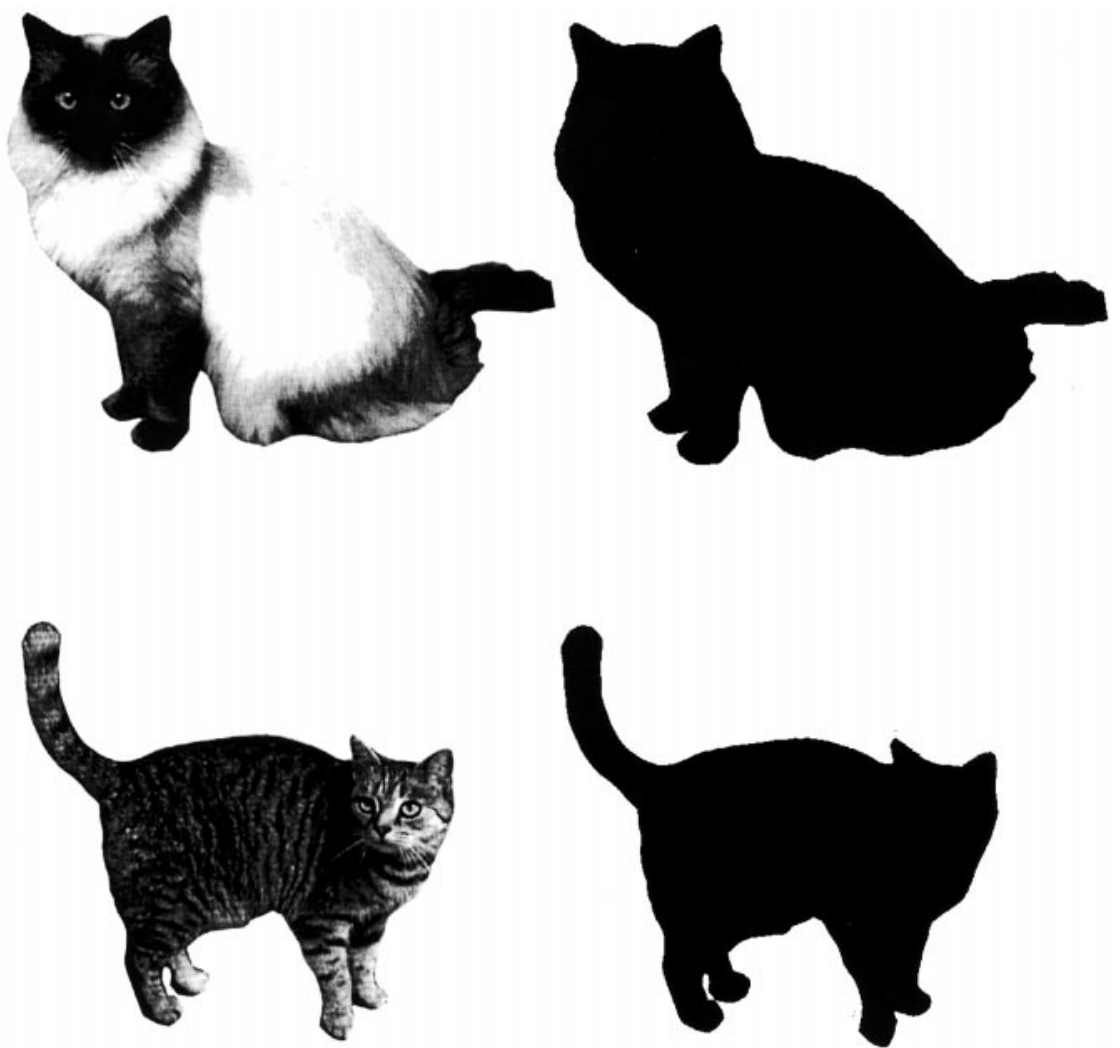

FIG. 1. Examples of two cat photographs (black-and-white) and their corresponding silhouettes. The photographs used in Quinn and Eimas (1996a) were in color.

Procedure. All infants in each experiment underwent the following general procedure. They were brought to the laboratory by a parent and seated in a reclining position on the parent's lap. There were two experimenters, both of whom were naive to the hypotheses under investigation. The first experimenter positioned the apparatus so that the midline of the infant's head was aligned with the midline of the display panel. When the display panel was open, the infant could see the experimenter from the midsection upward in addition to a portion of the room that was painted a light cream color. The experimenter selected the appropriate stimuli as previously determined for the forthcoming trial and loaded them into the compartments of the display panel from a nearby table. The experimenter then elicited the infant's attention and closed the panel, thereby exposing the stimuli to the infant. The parent was unable to see the stimuli.

During each trial, the first experimenter observed the infant through the small peephole and recorded visual fixations to the left and right stimuli by means of two $605 \mathrm{XE}$ Accusplit electronic stop watches, one of which was held in each 

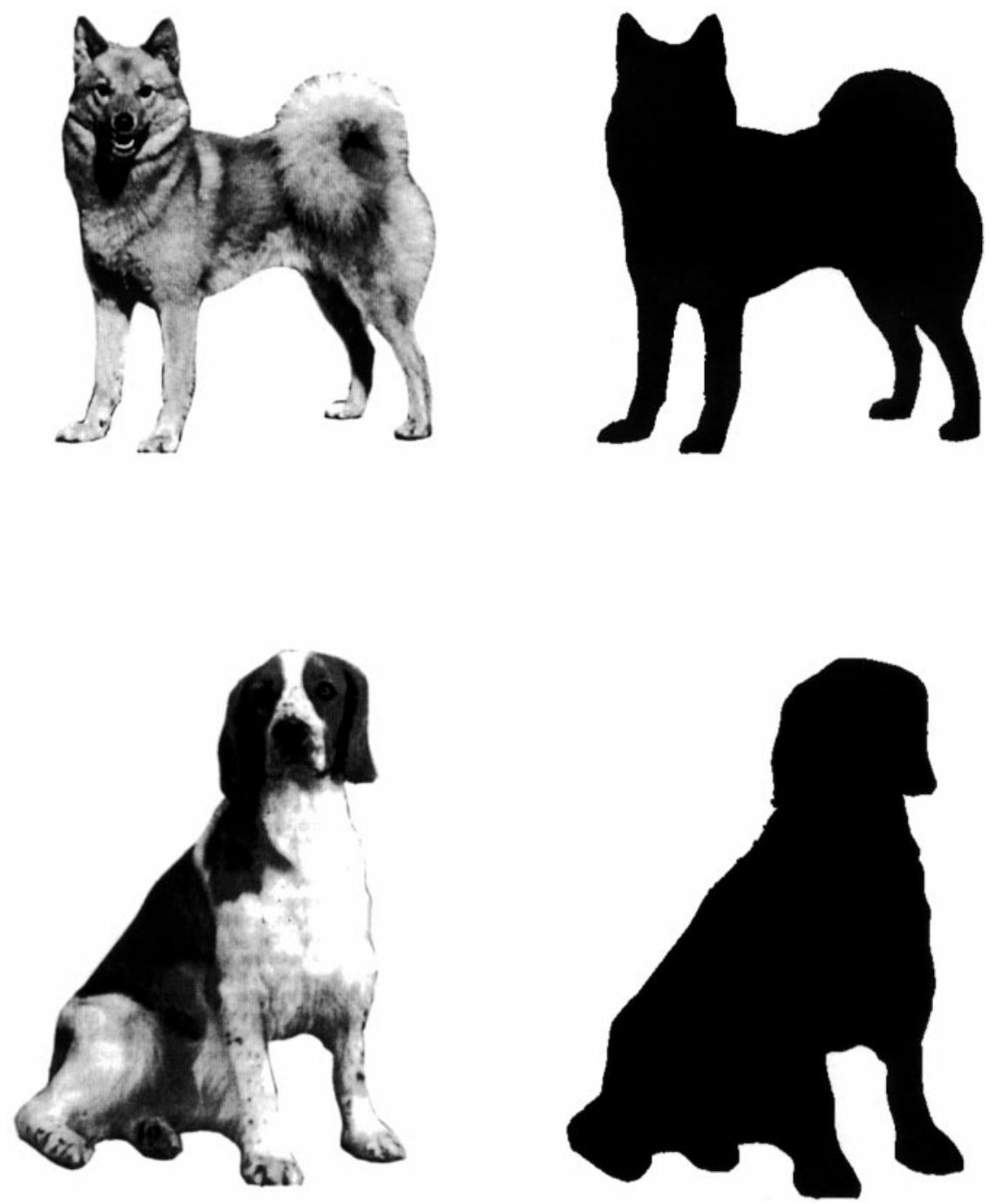

FIG. 2. Examples of two dog photographs (black-and-white) and their corresponding silhouettes. The photographs used in Quinn and Eimas (1996a) were in color.

hand. Interobserver reliability, as determined by comparing the looking times measured by the experimenter using the center peephole, and additional observers using peepholes to the left of the left stimulus compartment and to the right of right stimulus compartment, averaged 0.92. Between trials, the experimenter opened the panel, changed the stimuli, obtained the infant's attention and centered his or her gaze, and finally closed the panel. The second experimenter timed the individual trials and indicated the end of a familiarization trial. After familiariza- 
tion, the first and second experimenters changed places for the test trials. The experimenter who presented stimuli and measured the infant's fixations during familiarization now measured trial duration and signaled the end of each test trial, whereas the second experimenter presented the test stimuli and measured the infant's fixations. The two experimenters changed roles across infants. The second experimenter was always naive with respect to the familiar category (Experiments 1 and 3) or single familiar stimulus that was presented when the discriminability of two exemplars was measured (Experiments 2 and 4).

In Experiment 1, 24 infants were familiarized with 12 silhouettes of cats and 24 infants with 12 silhouettes of dogs. The 12 stimuli were randomly selected separately for each infant from each set of 18 stimuli and presented at the rate of two per trial for six 15-s trials. Which two of the 12 stimuli were shown on a given trial was likewise randomly determined. Immediately after familiarization and without interrupting the procedure, two 10-s test trials were presented. The stimuli for these trials paired a novel cat silhouette with a novel dog silhouette. The test stimuli were randomly selected from the remaining unfamiliar stimuli and were different for each infant. The left-right positioning of the test trial stimuli was counterbalanced across infants on the first test trial and reversed on the second test trial.

\section{Results and Discussion}

Familiarization trials. Individual looking times were summed over the two stimuli that were presented on each trial and then averaged across the first three and last three trials. Mean looking times in seconds and standard deviations were $9.66(S D=3.59)$ and $8.71(S D=3.68)$ for cats and $10.52(S D=3.00)$ and 9.50 $(S D=3.65)$ for dogs. An analysis of variance, Familiar Category (Cats vs Dogs) by Trials (1-3 vs $4-6$ ), was performed on the individual looking times and revealed a reliable effect of trials, $F(1,46)=8.00, p<.007$. All other effects were not significant, $F(1,46)<1.00, p>.20$, in each case. The decrement in looking time across trials, which has not always occurred with the colored pictures (e.g., Quinn et al., 1993), may have been facilitated in the present case by the absence of color and facial features (cf. Quinn \& Eimas, 1996a), making the pictures less attractive across trials.

Preference test trials. The looking time of each infant across the two test trials to the novel stimulus from the novel category was divided by the looking time to both test stimuli and converted to a percentage score by multiplying by 100 . Mean novelty preference scores were 58.62\% $(S D=18.31)$ for infants familiarized with cats and $57.44 \%(S D=15.66)$ for infants familiarized with dogs, both of which were reliably higher than chance, that is, 50\%,t(23)>2.30, $p<.025$, one-tailed, in each case. The difference between the two groups was not significant, $t(46)=0.24, p>.20$, two-tailed. In addition, 19 of 24 infants familiarized with cats displayed novel category preference scores for dogs that were greater than $50 \%(p<.05)$, and 17 of 24 infants familiarized with dogs displayed novel category preference scores that were greater than $50 \%(p<.05)$. 
In sum, there was evidence for categorization of the silhouettes of cats and dogs into separate representations, even though the stimuli lacked internal facial features, which are sufficient cues for categorization (Quinn \& Eimas, 1996a; Spencer et al., 1997), and the animals' typical variation in coloring. Despite this result, to conclude that categorization has occurred is premature. It is necessary to be certain that individual exemplars within each of the two categories were discriminably different. This was the case for the original naturally colored photographs (Quinn et al., 1993), but whether it was true for the silhouettes was determined in Experiment 2.

\section{EXPERIMENT 2}

Each infant was familiarized with a single cat (or dog) silhouette for one 15-s trial and then given a preference test that paired the familiar cat (or dog) silhouette with a novel cat (or dog) silhouette. A single familiarization trial was used so that the amount of potential familiarization time for an individual exemplar matched that provided in the categorization task of Experiment 1 (cf. BehlChadha \& Eimas, 1995).

\section{Method}

Participants. The infants were 243 - to 4-month-olds $(M=3.37$ months; $S D=$ .33 months). Thirteen infants were males and 11 were females. Eight additional infants failed to complete the procedure due to fussiness $(n=4)$, failure to look at both stimuli on the test trials $(n=2)$, and experimenter error $(n=2)$.

Procedure. Each infant was presented with two copies of a single, randomly selected cat $(n=12)$ or $\operatorname{dog}(n=12)$ silhouette for one 15 -s familiarization trial. Two 10-s test trials were then administered in which the familiar stimulus was paired with a novel stimulus from the same category. The novel animal silhouette was randomly selected and, like the familiar stimulus, differed for each infant. The left-right position of the novel animal was counterbalanced across infants on the first test trial and reversed on the second trial.

\section{Results and Discussion}

Mean looking time to the familiar cat was $9.57 \mathrm{~s}(S D=3.59)$ and $9.87 \mathrm{~s}(S D=$ 4.30) for the familiar dog. The difference in mean looking times was not significant, $t(22)=-0.35, p>.20$, two-tailed. Mean preference scores were $59.42 \%$ $(S D=20.66)$ for the novel cat and $61.86 \%(S D=20.91)$ for the novel dog. Both scores differed significantly from chance, i.e., $50 \%, t(11)>2.23, p<.025$, onetailed, in each case. The two preference scores did not differ reliably from each other, $t(22)=-0.37, p>.20$, two-tailed. In addition, 9 of 12 infants in both conditions showed a novelty preference score that was greater than $50 \%(p=.05)$. Given that both novelty scores were reliably greater than expected by chance, it is clear that individual exemplars from within each of the two categories were discriminably different from each other, just as they were previously when colored photographs as opposed to silhouettes were used (Quinn et al., 1993). It would 
thus appear that Experiment 1 did indeed show evidence of categorization on the basis of the shape information available from silhouettes.

An inference of categorization requires not only that the individual exemplars from a specific category be discriminably different, but also that spontaneous preferences favoring the exemplars of one or the other category not be present or at least not account for the entire novelty preference effect. Given the design of Experiment 1 in which members of both categories were familiar for one group of participants and novel for the other and that participants in both groups showed reliable novelty preference scores that did not differ from each other, one can safely infer that spontaneous preferences were not operative in Experiment 1. Thus, we can conclude that the silhouettes of cats and dogs were formed into categorical representations, just as was the case with their natural-looking counterparts in our previous study (Quinn et al., 1993).

\section{EXPERIMENT 3}

The question of concern for Experiment 3 was what information the infants used during formation of the categorical representations of the silhouettes in Experiment 1. Experiment 1 was thus repeated, but in this case with half of the infants presented with Head Only stimuli and the other half with Body Only stimuli, during both familiarization and test trials. As noted in the introduction, earlier work with colored photographs (Quinn \& Eimas, 1996a) had shown that infants of the same age used information that centered in the region of the head in forming categorical representations. Infants who saw the entire body alone, that is, the body without the head region, did not form categorical representations. The earlier work had also shown that infants could use the enlarged cat and dog heads in which the internal facial features (i.e., eyes, nose, and mouth) were occluded to categorically differentiate between the two species (Quinn \& Eimas, 1996a). However, internal regions of the head surrounding these features were visible and revealed species-typical coloring, markings, and texture. At issue here is whether external information in the head region, without the configural information provided by the eyes, nose, and mouth, and without the internal speciestypical coloring, markings, and texture of the head, would again be sufficient for category formation. In the present case, the only information available was shape as defined by the external contour of the head. Thus, only in the current experiment with silhouettes were we able to completely isolate the contribution of external contour and global shape of the head independently of characteristic mammal facial attributes.

\section{Method}

Participants. There were 48 infants 3 and 4 months of age $(M=3.30$ months; $S D=.37$ months). Twenty-six of the infants were females and 22 were males. An additional 7 infants did not complete the experiment because of fussing $(n=2)$, failure to look at both test stimuli $(n=3)$, and parental or sibling interference $(n=2)$. 
Stimuli. Stimuli were the silhouettes used in Experiments 1 and 2, and following the stimulus preparation used with the colored images in Quinn and Eimas (1996a), they were altered by placing rectangular pieces of gray construction paper over the body when the heads were presented and occluding the heads with similar but smaller pieces of gray construction paper when the bodies were presented.

Procedure. Twenty-four infants were randomly assigned to each of two groups: Head Only and Body Only. In each group, half of the infants were familiarized and tested with heads or bodies of dogs and half with heads or bodies of cats. In all other respects the procedural details were identical to those used in Experiment 1.

\section{Results and Discussion}

Familiarization trials. Mean looking times for the first and second trial blocks were $8.63 \mathrm{~s}(S D=3.11)$ and $7.60 \mathrm{~s}(S D=3.03)$ for the Body Only stimuli and $8.34 \mathrm{~s}(S D=3.08)$ and $7.65 \mathrm{~s}(S D=3.36)$ for the Head Only stimuli. An analysis of variance, Experimental Condition (Head Only vs Body Only) $\times$ Familiar Category (Cats vs Dogs) $\times$ Trials (1-3 vs 4-6), revealed only a reliable effect of trials, $F(1,44)=4.77, \mathrm{p}<.05$, with looking time decreasing from the first to the second block. All other $F$ values were less than 1.95, $p>.15$.

Preference test trials. Mean novelty preference scores were $51.02 \%$ ( $S D=$ 24.04) for the Body Only condition and 57.67\% ( $S D=18.45)$ for the Head Only condition. Only the Head Only mean differed reliably from chance, $t(23)=2.03$, $p<.05$, one-tailed. Within each condition, the mean novel category preference scores for infants familiarized with cats versus dogs did not differ, $t(22)<1.30$, $p>.20$, two-tailed, in both cases. In terms of individual preference scores, only 13 of 24 infants in the Body Only condition displayed a novel category preference score that was greater than $50 \%(p>.05)$, whereas 17 of 24 infants in the Head Only condition showed a novel category preference score that was greater than $50 \%(p<.05)$.

As shown previously (Quinn \& Eimas, 1996a), the infants formed categorical representations only when the stimuli consisted of the head regions of the dogs and cats. The new data indicate that the bounding contour information in this region, independently of internal facial features or species typical markings, is sufficient for the formation of categorical representations. The results also help to clarify the earlier finding that infants could use the enlarged cat and dog heads in which the internal facial features were occluded to categorically differentiate between the two species (Quinn \& Eimas, 1996a). The previous result had left open the possibility that the species-typical coloring and markings of the head that were detectable outside of the internal feature region could have facilitated the formation of the categorical representations.

It is of additional interest to note that, at least in the case of the natural pictures, adults readily classified the bodies alone as cats and dogs (Quinn \& Eimas, 1996a), and presentation of the 36 silhouettes of the bodies in a random order to 
5 adults in a forced-choice cat versus dog identification task produced nearly the same results- the exemplars were classifiable as dogs or cats, with an average of only 4.8 errors $(S D=3.1)$ per adult. Why infants make use of only the head region remains open to question (but see commentary under General Discussion) as does when the information carried by the bodies becomes relevant to infants or young children.

Before proceeding to the General Discussion, it is first necessary to establish whether the infants can discriminate between the individual Head Only and Body Only stimuli within the cat and dog categories. Positive evidence for discrimination of the Head Only stimuli within the cat and dog categories would imply that the infants in the Head Only condition of Experiment 3 formed representations of discriminably different cat or dog heads. Positive evidence for discrimination of the Body Only stimuli within the cat and dog categories would imply that the infants are capable of discriminating individual cat (or dog) bodies from one another even though the infants did not display the ability to form representations for cat (or dog) bodies which were sufficiently structured so as to exclude instances of dog (or cat) bodies. Just as Experiment 2 was conducted as the within-category discrimination control for Experiment 1, Experiment 4 was conducted as the within-category discrimination control for Experiment 3.

\section{EXPERIMENT 4}

Because categorization refers to equivalent responding to discriminably different entities, Experiment 4 was conducted to determine if 3- and 4-month-old infants could discriminate within the cat and dog stimulus categories used in Experiment 3. Each infant was familiarized with either the Head Only or Body Only version of a single cat (or dog) silhouette and then tested with the familiar cat (or dog) silhouette paired with a novel cat (or dog) silhouette. As was the case in Experiment 2, a single 15-s familiarization trial was used so that the amount of potential familiarization time for an individual exemplar matched that provided in the categorization task of Experiment 3.

\section{Method}

Participants. The infants were 243 - to 4-month-olds ( $M=3.61$ months; $S D=$ .33 months). Thirteen infants were males and 11 were females. One additional infant failed to complete the procedure due to fussiness.

Procedure. Twelve infants were randomly assigned to each experimental group: Head Only and Body Only. As in Experiment 3, infants in the Head Only group were tested with cat (or dog) heads alone (body parts were occluded), whereas those in the Body Only group were tested with cat (or dog) bodies alone (heads were occluded). Each infant was presented with two identical copies of a single randomly selected cat (or dog) part for one 15-s familiarization trial. Two 10-s test trials were then administered in which the familiar stimulus part was paired with a novel stimulus part from the same category. The novel animal silhouette part was again randomly selected and like the familiar stimulus part differed for each 
infant. The left-right position of the novel stimulus part was counterbalanced across infants on the first test trial and reversed on the second trial.

\section{Results and Discussion}

Familiarization trial. An analysis of variance of the individual looking times, with Experimental Condition (Head Only vs Body Only) and Familiar Stimulus (Cat vs Dog) as between-subjects factors, revealed only an effect of Experimental Condition, $F(1,20)=6.00, p=.022$. This effect reflected a significant difference in mean looking time to the Head Only stimuli $(M=8.17 \mathrm{~s}, S D=2.24)$ and the Body Only Stimuli $(M=11.15 \mathrm{~s}, S D=3.53)$ during the single familiarization trial. The difference was most likely attributable to the greater amount of black contour information present in the animal bodies relative to the animal heads.

Preference test trials. An analysis of variance of the individual novelty preference scores, with Experimental Condition and Familiar Stimulus as between-subjects factors, did not yield any significant effects, $F(1,20)<1.07$, p $>.30$, in each instance. Mean novelty preference scores were $65.58 \%(S D=10.80)$ for the Head Only group and $63.18 \%(S D=18.46)$ for the Body Only group. Both scores differed significantly from chance, that is, 50\%, $t(11)>2.46, p<.025$, one-tailed, in each case. In addition, 12 of 12 infants in the Head Only group showed individual novelty preference scores that were greater than $50 \%$ ( $p<$ .05 ), and 10 of 12 infants in the Body Only group displayed individual novelty preference scores that were greater than $50 \%(p<.05)$. Given that both novelty scores were reliably greater than expected by chance, it is clear that individual Head Only and Body Only stimuli from within the cat and dog categories were discriminably different from each other, just as they were previously when colored photographs as opposed to silhouettes were used (Quinn \& Eimas, 1996a). The Head Only result indicates that the infants in Experiment 3 did indeed show evidence of categorization compatible with a mechanism sensitive to the shape information available in the silhouettes of the heads. The Body Only finding indicates that while infants can discriminate one cat (or dog) body from another, they did not use this information to form a categorical representation for cats (or dogs) that excludes dogs (or cats) in Experiment 3.

\section{GENERAL DISCUSSION}

Four experiments were conducted to examine whether young infants could categorize cat and dog stimuli on the basis of silhouette information. In Experiment 1, 3- to 4-month-old infants familiarized with cat or dog silhouettes preferred a silhouette from the novel category over a novel silhouette from the familiar category. Experiment 2 showed that the infants were able to discriminate between different silhouettes from the same category; for example, after familiarization with one cat (or dog) silhouette, a novel cat (or dog) silhouette was preferred to the familiar silhouette. Experiment 3 revealed that the infants were able to categorically separate the cats and dogs on the basis of contour information from the head region. Subsequent to familiarization with silhouettes of cat or dog heads (the 
body information was covered), the infants preferred a head silhouette from the novel category over a novel head silhouette from the familiar category. In contrast, infants familiarized with silhouettes of cat or dog bodies (the head information was covered) did not prefer a body silhouette from the novel category over a novel body silhouette from the familiar category. In Experiment 4, individual cat (or dog) head silhouettes were shown to be discriminable from each other, as were individual cat (or dog) body silhouettes.

The evidence from Experiments 1 and 2, taken in conjunction with previous findings (Quinn et al., 1993), suggests that 3- and 4-month-olds can represent discriminably different cat and dog stimuli as separate categories on the basis of silhouette information as well as perform this differentiation with the actual photographs that contain color and internal feature information. The performance of the young infants corresponds well with adults, who likewise can identify objects at the basic level on the basis of line drawings (Biederman \& Ju, 1988) or silhouettes (Hayward, 1998; Rosch et al., 1976) as well as identify colored photographs of the objects (Murphy \& Smith, 1982). Consistent with these findings are the results of computational simulations that measure silhouette similarity between objects and indicate that there is sufficient information in silhouettes to place objects in their correct basic-level categories (Cutzu \& Tarr, 1997; Gdalyahu \& Weinshall, 1996; Ullman, 1996). It is also noteworthy that children display a bias toward extending novel nouns on the basis of global shape information (Landau, Smith, \& Jones, 1988). Throughout development then, shape information, as specified by an object's bounding contour, may hold a privileged status in the representation of objects.

The data of Experiments 3 and 4 add to our understanding of early categorization by showing that young infants can use bounding contour information from the head silhouettes, but not the body silhouettes, to form individuated representations for the cat and dog stimuli. The findings parallel evidence obtained with young infants performing with the actual head and body photographs of the cat and dog stimuli (Quinn \& Eimas, 1996a; Spencer et al., 1997). It is interesting to comment on the possible processing systems that could accommodate these data. The advantage for the head over the body region, demonstrated for both the actual photographs and silhouettes, is consistent with a domain-general processing account. Information detected from the head may simply be more diagnostic of cat versus dog category membership (i.e., heads are more different than bodies) and thus more readily detected and utilized than information arising from the body, at least for infants (see also the neural networks of Quinn \& Johnson, 1997, for simulation results consistent with the empirical evidence).

The data from Experiment 3 further imply that a face-specific processing mechanism like CONSPEC is not absolutely necessary to account for the categorical differentiation of cats and dogs at least at this age. If CONSPEC as described by Johnson and Morton (1991) was needed to accomplish the categorical separation of the two species, then one would not have expected a head over body advantage with the silhouette stimuli. This expectation is based on the fact 
that the domain-specific CONSPEC mechanism is presumed to be activated by internal and configural facial information not present in the silhouettes. However, as noted in the introduction, if CONSPEC were reformulated to explicitly include bounding contour information from the head region, then it would remain a viable means of explanation for the present findings.

Another possible processing scheme is one in which a domain-specific bias to attend to heads (via a CONSPEC-like mechanism that is activated as well by the external contour of the head) combines with a domain-general learning mechanism that computes the highly diagnostic shape of the head. By this scheme, domain-specific biases and domain-general learning mechanisms may play complementary roles in the formation of perceptual category representations by young infants. Indeed, biases plus general learning mechanisms might well be necessary for the start-up of categorization in a variety of domains.

A final point is that the role that early perceptually based categorical representations play in support of higher level concepts remains controversial (Madole \& Oakes, 1999; Mandler \& McDonough, 1998; Quinn \& Eimas, 1997; Xu \& Carey, 1996). Clearly, children and adults come to know animals as distinct "kinds" through information that is not available to young infants (e.g., that dogs make good pets, possess dog DNA, and give birth to puppies). Our view is that the early parsing of the world based on surface features, given its validity to reality, would permit the linkage of this more conceptual knowledge with the early perceptually based categorical representations formed by young infants. By this view, young infants' categorical representations may form the "primitive base" or "bottom layer" from which adult conceptions of object kinds are constructed.

\section{REFERENCES}

Anderson, J. A. (1995). Introduction to neural networks. (pp. 433-462). Cambridge, MA: MIT Press.

Balaban, M. T., \& Waxman, S. R. (1997). Do words facilitate object categorization in 9-month-old infants? Journal of Experimental Child Psychology, 64, 3-26.

Behl-Chadha, G., \& Eimas, P. D. (1995). Infant categorization of left-right spatial relations. British Journal of Developmental Psychology, 13, 69-79.

Biederman, I., \& Ju, G. (1988). Surface versus edge-based determinants of visual recognition. Cognitive Psychology, 20, 38-64.

Carey, S. (1985). Conceptual change in childhood. Cambridge, MA: MIT Press.

Cutzu, F., \& Tarr, M. J. (1997). The representation of three-dimensional object similarity in human vision. In SPIE Proceedings From Electronic Imaging: Human Vision \& Electronic Imaging II, 3016 (pp. 460-471). San Jose, CA: SPIE.

Edelman, G. M. (1987). Neural Darwinism. New York: Basic Books.

Eimas, P.D., \& Quinn, P.C. (1994). Studies on the formation of perceptually based basic-level categories in young infants. Child Development, 65, 903-917.

Eimas, P.D., Quinn, P.C., \& Cowan, P. (1994). Development of exclusivity in perceptually based categories of young infants. Journal of Experimental Child Psychology, 58, 418-431.

Fantz, R. L. (1964). Visual experience in infants: Decreased attention to familiar patterns relative to novel ones. Science, 164, 668-670.

Gdalyahu, Y., \& Weinshall, D. (1996, June). Measures for silhouettes resemblance and representative silhouettes of curved objects. Paper presented at the Fourth European Conference on Computer Vision, Cambridge, UK. 
Gelman, S. A. (1996). Concepts and theories. In R. Gelman \& T. Kit-Fong Au (Eds.), Perceptual and cognitive development (pp. 117-150). San Diego: Academic Press.

Goldstone, R. L., \& Barsalou, L. W. (1998). Reuniting perception and conception. Cognition, 65, 231-262.

Hayward, W. G. (1998). Effects of outline shape in object recognition. Journal of Experimental Psychology: Human Perception and Performance, 24, 427-440.

Johnson, M. H., \& Morton, J. (1991). Biology and cognitive development: The case of face recognition. Cambridge, MA: Blackwell.

Jones, S. S., \& Smith, L. B. (1993). The place of perception in children's concepts. Cognitive Development, 8, 113-139.

Keil, F. C. (1989). Concepts, kinds, and cognitive development. Cambridge, MA: MIT Press.

Kurbat, M. A., Smith, E. E., \& Medin, D. L. (1994). Categorization, typicality, and shape similarity. In Proceedings of the Sixteenth Annual Conference of the Cognitive Science Society. Hillsdale, NJ: Erlbaum.

Landau, B., Smith, L. B., \& Jones, S. S. (1988). The importance of shape in early lexical learning. Cognitive Development, 3, 299-321.

Madole, K. L., \& Oakes, L. M. (1999). Making sense of infant categorization: Stable processes and changing representations. Developmental Review, 19, 263-296.

Malt, B. C. (1995). Category coherence in cross-cultural perspective. Cognitive Psychology, 29, 85-148.

Malt, B. C., \& Smith, E. E. (1984). Correlated properties in natural categories. Journal of Verbal Learning \& Verbal Behavior, 23, 250-269.

Mandler, J. M., \& McDonough, L. (1993). Concept formation in infancy. Cognitive Development, 8, 291-318.

Mandler, J. M., \& McDonough, L. (1998). On developing a knowledge base in infancy. Developmental Psychology, 34, 1274-1288.

Marr, D. (1982). Vision. San Francisco: Freeman.

Millikan, R. G. (1998). A common structure for concepts of individuals, stuffs, and real kinds: More mama, more milk, and more mouse. Behavioral \& Brain Sciences, 21, 55-100.

Murphy, G. L. (1991). Parts in object concepts: Experiments with artificial categories. Memory \& Cognition, 19, 423-438.

Murphy, G. L., \& Smith, E. E. (1982). Basic-level superiority in picture categorization. Journal of Verbal Learning and Verbal Behavior, 21, 1-20.

Quinn, P. C., \& Eimas, P. D. (1996a). Perceptual cues that permit categorical differentiation of animal species by infants. Journal of Experimental Child Psychology, 63, 189-211.

Quinn, P. C., \& Eimas, P. D. (1996b). Perceptual organization and categorization in young infants. In C. Rovee-Collier \& L. P. Lipsitt (Eds.), Advances in infancy research (Vol. 10, pp. 1-36). Norwood, NJ: Ablex.

Quinn, P. C., \& Eimas, P. D. (1997). A reexamination of the perceptual-to-conceptual shift in mental representations. Review of General Psychology, 1, 271-287.

Quinn, P. C., \& Eimas, P. D. (1998). Evidence for a global categorical representation of humans by young infants. Journal of Experimental Child Psychology, 69, 151-174.

Quinn, P. C., Eimas, P. D., \& Rosenkrantz, S. L. (1993). Evidence for representations of perceptually similar natural categories by 3-month-old and 4-month-old infants. Perception, 22, 463-475.

Quinn, P. C., \& Johnson, M. H. (1997). The emergence of perceptual category representations in young infants: A connectionist analysis. Journal of Experimental Child Psychology, 66, 236-263.

Rakison, D. H., \& Butterworth, G. E. (1998). Infants' use of object parts in early categorization. Developmental Psychology, 34, 49-62.

Rosch, E., Mervis, C. B., Gray, W. D., Johnson, D. M., \& Boyes-Braem, P. (1976). Basic objects in natural categories. Cognitive Psychology, 8, 382-439.

Schyns, P. G., Goldstone, R. L., \& Thibaut, J. P. (1998). The development of features in object concepts. Behavioral \& Brain Sciences, 21, 1-54. 
Slater, A. M. (1995). Visual perception and memory at birth. In C. Rovee-Collier \& L. P. Lipsitt (Eds.), Advances in infancy research (Vol. 9, pp. 107-162). Norwood, NJ: Ablex.

Spencer, J., Quinn, P. C., Johnson, M. H., \& Karmiloff-Smith, A. (1997). Heads you win, tails you lose: Evidence for young infants categorizing mammals by head and facial attributes (Special Issue: Perceptual Development). Early Development \& Parenting, 6, 113-126.

Thelen, E., \& Smith, L. B. (1994). A dynamic systems approach to the development of cognition and action. Cambridge, MA: MIT Press.

Tversky, B., \& Hemenway, K. (1984). Objects, parts, and categories. Journal of Experimental Psychology: General, 113, 169-193.

Ullman S. (1996). High-level vision: Object recognition and visual cognition. Cambridge, MA: MIT Press.

Waxman, S. R., \& Markow, D. B. (1995). Words as invitations to form categories: Evidence from 12to 13-month-old infants. Cognitive Psychology, 29, 257-302.

Wellman, H. (1990). Children's theories of mind. Cambridge, MA: MIT Press/Bradford Books.

Xu, F., \& Carey, S. (1996). Infants' metaphysics: The case of numerical identity. Cognitive Psychology, 30, 111-153.

Received October 19, 1999; revised June 29, 2000 